\title{
Infrared Colloidal Quantum Dot Photovoltaics via Coupling Enhancement and Agglomeration Suppression
}

\author{
Alexander H. Ip, Amirreza Kiani, Illan J. Kramer, \\ Oleksandr Voznyy, Hamidreza F. Movahed, Larissa Levina, \\ Michael M. Adachi, Sjoerd Hoogland, and Edward H. Sargent
}

Version Post-Print/Accepted Manuscript

Citation Ip, A. H., Kiani, A., Kramer, I. J., Voznyy, O., Movahed, H. F., Levina, (published version) L., Adachi, M. M., Hoogland, S., and Sargent, E. H. (2015). Infrared colloidal quantum dot Photovoltaics via coupling enhancement and agglomeration suppression. ACS Nano, 9(9), 8833-8842.

doi:10.1021/acsnano.5b02164

\begin{abstract}
Publisher's Statement This document is the Accepted Manuscript version of a Published Work that appeared in final form in ACS Nano, copyright (C)American Chemical Society after peer review and technical editing by the publisher. To access the final edited and published work see http://dx.doi.org/10.1021/acsnano.5b02164.
\end{abstract}

How to cite TSpace items

Always cite the published version, so the author(s) will receive recognition through services that track citation counts, e.g. Scopus. If you need to cite the page number of the TSpace version (original manuscript or accepted manuscript) because you cannot access the published version, then cite the TSpace version in addition to the published version using the permanent URI (handle) found on the record page. 


\title{
Infrared Colloidal Quantum Dot
} Photovoltaics via Coupling

\section{Enhancement and Agglomeration Suppression}

\author{
Alexander H. Ip, ${ }^{\ddagger}$ Amirreza Kiani, ${ }^{\ddagger}$ Illan J. Kramer, ${ }^{\ddagger}$ Oleksandr Voznyy, Hamidreza F. Movahed, Larissa Levina, \\ Michael M. Adachi, Sjoerd Hoogland, and Edward H. Sargent* \\ Department of Electrical and Computer Engineering, University of Toronto, 10 King's College Road, Toronto, Ontario M5S 3G4, Canada. ${ }^{\ddagger}$ A. H. Ip, A. Kiani, and \\ I. J. Kramer contributed equally.
}

\begin{abstract}
Single-junction solar cells, such as those based on silicon, perovskites, and colloidal quantum dots, fail to utilize a significant portion of the solar spectrum because they are unable to absorb photons having energy lower than their respective band gap. Here we explore through modeling and experiment the potential for colloidal quantum dots (CQDs) to augment the performance of solar cells by harnessing transmitted light in the infrared. Through detailed balance modeling, we identify the CQD band gap that is best able to augment wafer-based, thin-film, and also solution-
\end{abstract}
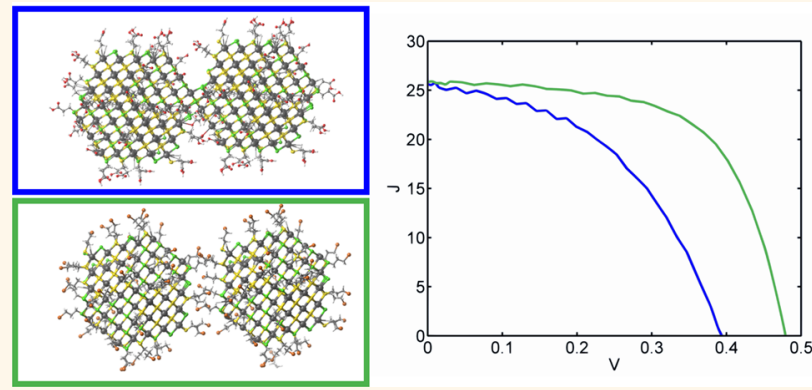
processed photovoltaic (PV) materials. The required quantum dots, with an excitonic peak at $1.3 \mu \mathrm{m}$, have not previously been studied in depth for solar performance. Using computational studies we find that a new ligand scheme distinct from that employed in better-explored $0.95 \mu \mathrm{m}$ band gap PbS CQDs is necessary; only via the solution-phase application of a short bromothiol can we prevent dot fusion during ensuing solid-state film treatments and simultaneously offer a high valence band-edge density of states to enhance hole transport. Photoluminescence spectra and transient studies confirm the desired narrowed emission peaks and reduced surface-trap-associated decay. Electronic characterization reveals that only through the use of the bromothiol ligands is strong hole transport retained. The films, when used to make PV devices, achieve the highest AM1.5 power conversion efficiency yet reported in a solution-processed material having a sub-1 eV band gap.

KEYWORDS: colloidal quantum dots $\cdot$ photovoltaics $\cdot$ infrared-absorbing solar cell $\cdot$ small band gap

$\int$ ilicon solar cells dominate solar photovoltaics (PV), ${ }^{1}$ with laboratory power conversion efficiencies (PCEs) that reach $25 \%{ }^{2}$ Thin-film CdTe and CulnGaSe (CIGS) cells have also demonstrated high efficiencies and are commercially available. Emerging solution-processed PV materials such as polymers ${ }^{3}$ and dyes ${ }^{4}$ have been developed as lower cost alternatives. Most recently, solution-processed perovskite solar cells have gained significant attention, and their certified efficiencies have rapidly increased to over $20 \% .^{5-8}$

Each of these cells leaves a large portion of the available solar spectrum untapped. Half of available photons reside in the infrared (IR), presenting a large opportunity to improve spectral utilization. ${ }^{9}$ Highefficiency III-V multijunction cells absorb this region ${ }^{10}$ but require costly wafer growth techniques and complex concentrator and tracking systems. Many low-cost solutionprocessed materials are transparent in this region, as even the narrowest-band-gap polymers and dyes have absorption onsets near 960 and $800 \mathrm{~nm}$ wavelengths, respectively, ${ }^{11,12}$ Highly efficient perovskite cells have absorption onsets at wavelengths shorter than $850 \mathrm{~nm}^{13,14}$

Colloidal quantum dots (CQDs) are a solution-processed material that can potentially overcome these limitations. CQDs have a band gap that is tunable via the quantum size effect. The absorption onset can be

$\begin{array}{lc}\text { *Address correspondence to } & 35 \\ \text { ted.sargent@utoronto.ca. } & 36 \\ \text { Received for review April 11, } 2015 & 37 \\ \text { and accepted August 12, 2015. } & 38 \\ \text { Published online } & 39 \\ \text { 10.1021/acsnano.5b02164 } & 40 \\ & 41 \\ \text { ๑) XXXX American Chemical Society } & 42 \\ \end{array}$


simply and precisely tuned across the solar spectrum. ${ }^{15}$

Because CQDs are synthesized in solution, they are amenable to rapid, large-area fabrication on lightweight, flexible substrates. ${ }^{16,17}$ Performance advances have focused on the passivation of trap states ${ }^{18-21}$ as well as development of device structures for optical absorption enhancement. ${ }^{22-24}$ The efficiency of CQD cells has thereby been advanced to certified efficiencies approaching $10 \% .^{25,26} \mathrm{In}$ addition to their promise as single-junction devices, CQD solar cells are attractive as a low-cost, IR-absorbing material that could be combined with other PV materials that lack infrared spectral response.

\section{RESULTS AND DISCUSSION}

We begin using a detailed balance approach to select a CQD band gap that will combine well with a variety of systems. Specifically, we considered crystalline silicon and methylammonium lead halide perovskite front absorbers due to their commercial ubiquity and rapid rise in efficiency, respectively. In Figure 1a bromide perovskite refers to $\mathrm{CH}_{3} \mathrm{NH}_{3} \mathrm{PbBr}_{3}$, while iodide perovskite refers to $\mathrm{CH}_{3} \mathrm{NH}_{3} \mathrm{Pbl}_{3}$. Figure $1 \mathrm{~b}$ shows the available short-circuit photocurrent density $\left(J_{S C}\right)$ for CQD cells as a function of CQD band gap for different front cells. Notably, there is sufficient light available to current-match to perovskites in a series-connected tandem, while the same is not true for the smallerband-gap silicon cell. There is also enough subgap photocurrent available to current-match with the best polymer triple-junction cells $\left(10 \mathrm{~mA} \mathrm{~cm}{ }^{-2}\right.$, not shown). ${ }^{27,28}$ The best-case open-circuit voltages $\left(V_{O C}\right)$ are shown in Figure 1c, and the theoretically achievable PCE is shown in Figure 1d. The band gap used for CQD solar cells with the best AM1.5 performance,

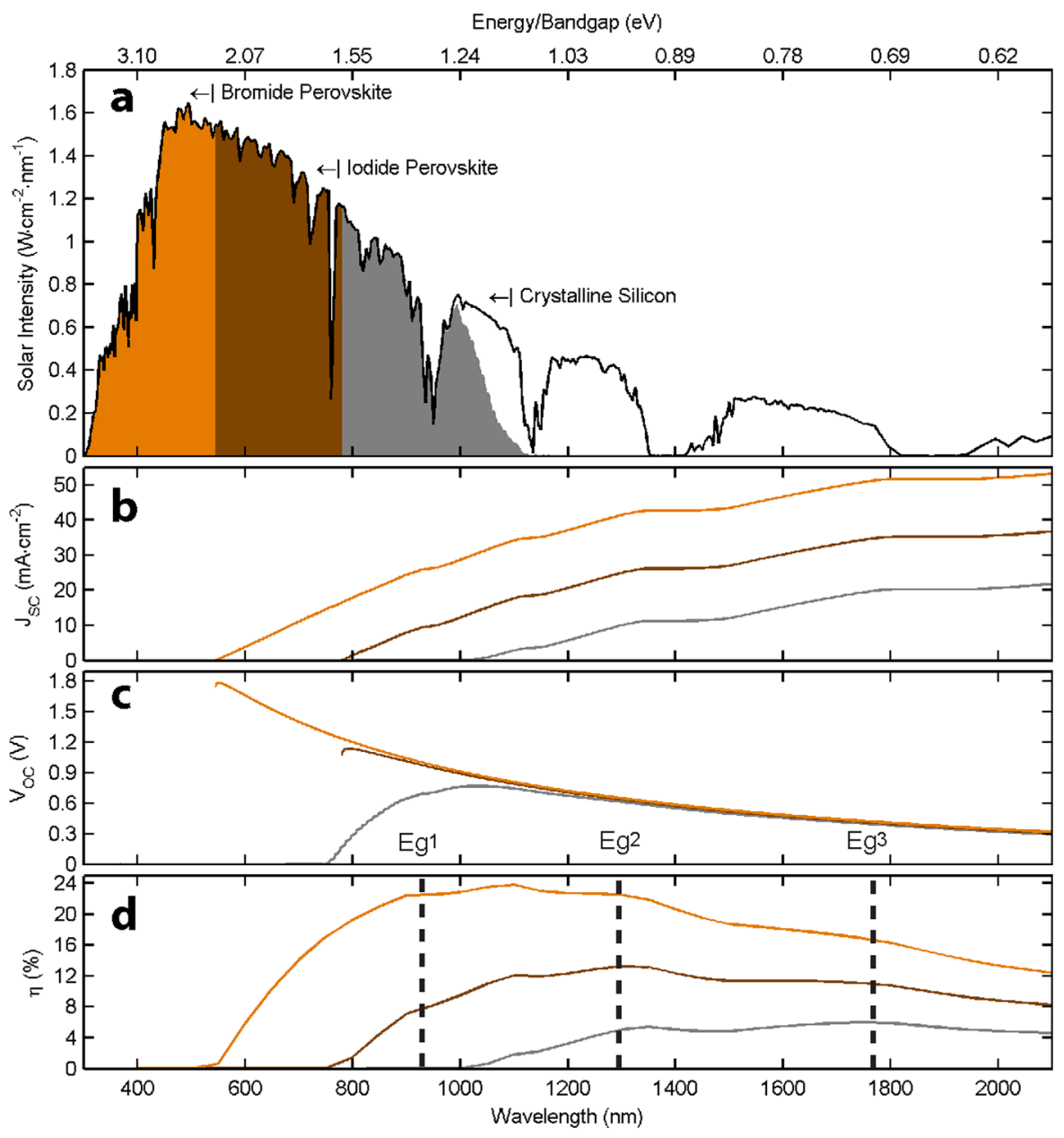

Figure 1. (a) Solar spectrum (black curve) with portions accessible to labeled material: orange is a lead bromide perovskite, orange + brown is a lead iodide perovskite, and orange + brown + gray is crystalline silicon. Everything to the right of each absorption edge represents the available solar spectrum for any subsequent cells. (b-d) Detailed balance simulations for solar cells accessing the solar spectrum transparent to the front cells identified in (a) according to the same color scheme including (b) short-circuit current density $\left(J_{\mathrm{SC}}\right)$, (c) open-circuit voltage $\left(V_{\mathrm{OC}}\right)$, and (d) power conversion efficiency (PCE). Band gaps of interest are marked here. $E_{g} 1$ corresponds to $\sim 950 \mathrm{~nm}$, the optimized single-junction band gap. $E_{g} 2$ corresponds to $1300 \mathrm{~nm}$, the edge of a broad peak for the lead bromide perovskite case, an absolute maximum for the lead iodide perovskite case, and a local maximum with little compromise to total efficiency in the crystalline silicon case. $E_{g} 3$ represents $1770 \mathrm{~nm}$, the optimal band gap for integration with silicon. 
$950 \mathrm{~nm}$, is marked as $E_{\mathrm{g}} 1$. The bromide perovskite allows a maximal additive CQD PCE with a band gap in the range 900 to $1300 \mathrm{~nm}$, with a peak of 22 additive points at $1100 \mathrm{~nm}$. The iodide perovskite peaks with approximately 12 power points for a $1300 \mathrm{~nm}$ band gap, noted in Figure $1 \mathrm{~d}$ as $E_{\mathrm{g}} 2$, with shoulders that extend from 1100 to $1800 \mathrm{~nm}$. The silicon case also shows a local maximum at about $1300 \mathrm{~nm}$ ( 6 additive power points), although the peak PCE is achieved at $1770 \mathrm{~nm}$, marked as $E_{\mathrm{g}} 3$. The broad maxima in these curves arise due to the dips in the AM1.5G spectrum caused by atmospheric water absorption of sunlight. Decreasing the band gap in these regions results in potential $V_{\text {OC }}$ loss with insufficient benefit to $J_{\mathrm{SC}}$.
In light of these findings, we focused on developing the $1.3 \mu \mathrm{m}$ band gap $\left(\mathrm{E}_{\mathrm{g}} 2\right) \mathrm{CQD}$ solid. We built our devices based on the fully developed depleted heterojunction structure. ${ }^{29}$ Figure 2 a shows a cross-sectional SEM image of the device, and Figure $2 \mathrm{~b}$ shows $2 \mathrm{D}$ and its corresponding 3D device schematics. First, a very thin layer of the polyethylenimine ethoxylated (PEIE) was spin-coated over the pre-etched indium - tin oxide (ITO)-coated glass substrate, after which we applied a $50 \mathrm{~nm}$ thick layer of $\mathrm{TiO}_{2}$ nanoparticles (NPs). ${ }^{30}$ The PEIE $/ \mathrm{TiO}_{2}$ stack formed low-temperature electron-acceptor layer. We have found that PEIE not only makes the charge collection at the electrode more efficient through lowering the barrier between ITO and $\mathrm{TiO}_{2} \mathrm{NPs}^{31}$ but also improves the adhesion

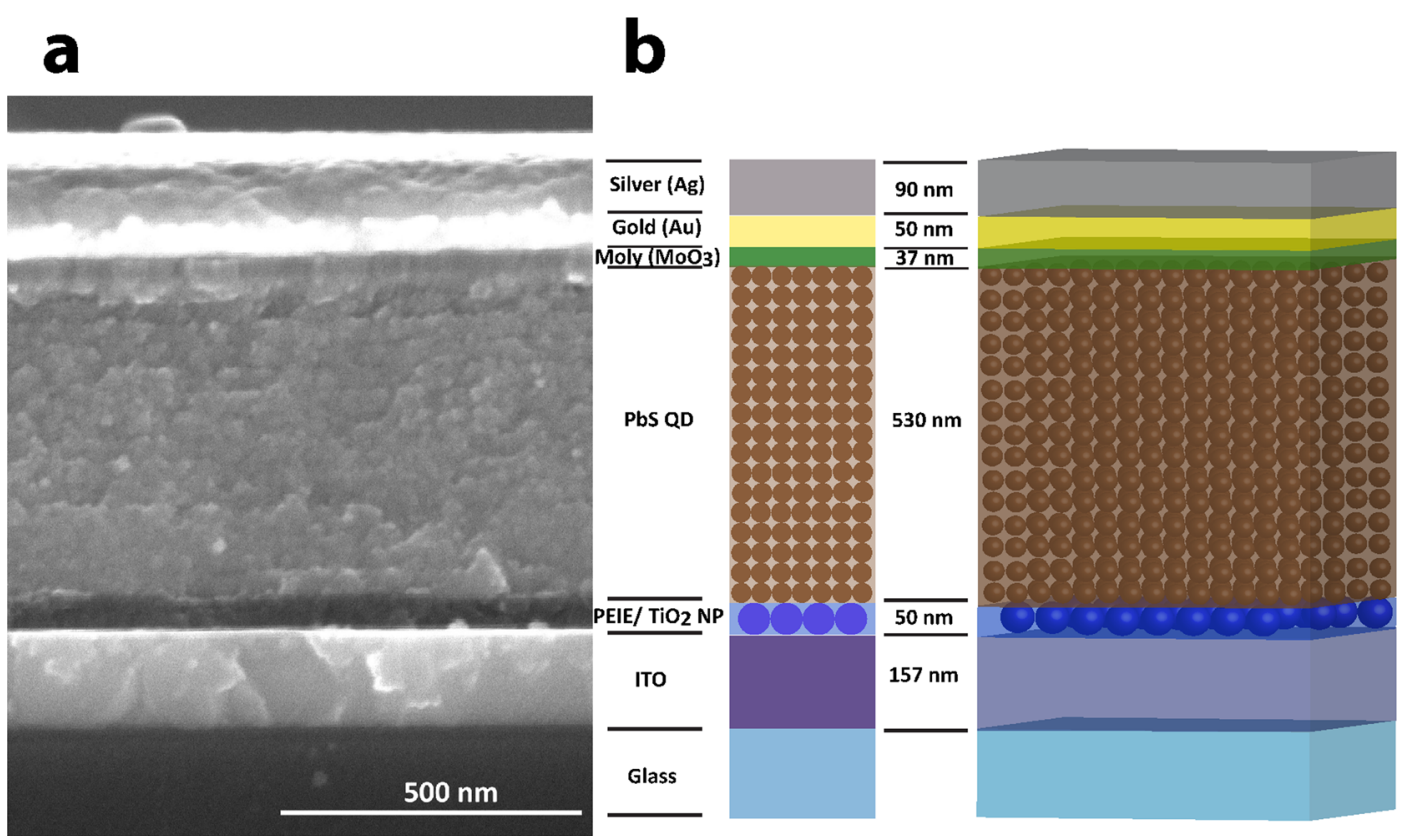

C

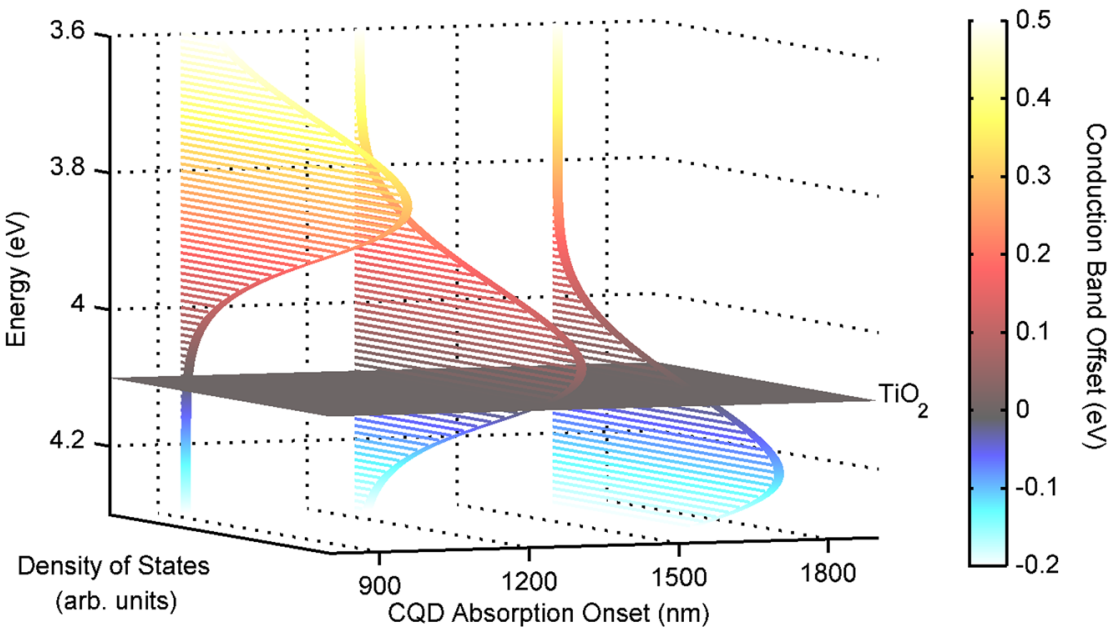

Figure 2. (a) Cross-sectional SEM image and (b) schematic of the depleted heterojunction CQD device. (c) Comparing TiO $\mathrm{C}_{2}$ and $\mathrm{PbS}$ CQD conduction band DOS with respect to CQD absorption onset at band gaps of interest. Positive values for the offset (toward yellow) indicate a favorable injection of electrons from the CQD conduction band into the $\mathrm{TiO}_{2}$ acceptor. Negative values for the offset (toward blue) mean there is a barrier for the electrons injecting into the $\mathrm{TiO}_{2}$. 
of the $\mathrm{TiO}_{2}$ and CQD layers substantially and hence yields a better quality film. A lead sulfide CQD film with a thickness of over $500 \mathrm{~nm}$ was used as the absorber. The thickness was optimized to maximize the absorption while allowing efficient charge collection.

Mercaptopropionic acid (MPA) was used to replace the poorly conductive, as-synthesized oleic acid (OA) ligands in a solid-state ligand exchange process. A thin layer of molybdenum oxide $\left(\mathrm{MoO}_{3}\right)$ was used as the hole collector. Gold and silver (140 nm combined) were used to form the top electrode. Figure $2 c$ shows schematically the conduction band-edge density of states (DOS) of the PbS CQD as a function of its absorption onset and compares it to the electron affinity of $\mathrm{TiO}_{2}{ }^{32}$ for the band gaps of interest from Figure $1 \mathrm{~d}$. For the optimal CQD single-junction band gap $\left(E_{g} 1\right)$, a favorable offset allows electron injection into the $\mathrm{TiO}_{2}$ even for a broad distribution of bandedge states, while at $\mathrm{E}_{\mathrm{g}} 3$ a large barrier between the CQD conduction band and that of $\mathrm{TiO}_{2}$ prevents electron transfer. The conduction band edge of optimal $E_{g} 2$ or $\sim 1300 \mathrm{~nm}$ PbS CQDs lies at the threshold of efficient electron injection: carriers in tail states would not be extracted.

We were therefore particularly attentive to possible agglomeration of CQDs, as these particles would have a narrower band gap and their conduction band would lie below that of the electron acceptor. As shown schematically in Figure 3a (top), exposing the surface of adjacent CQDs during solid-state ligand exchange can lead to fusion, creating larger nanoparticles with smaller band gaps. ${ }^{33-36}$ The fusing of nanocrystal surfaces has been used to affect the electronic properties of CQD solids and induce ordering in films, particularly in the case of large nanoparticles with well-defined facets. ${ }^{37-40}$ While transport is improved in fused dots, the quantum confinement is reduced and the sharpness of the band edge is lost. Solid-state ligand exchange is a dynamic equilibrium process in which methanol strips oleate from CQD surfaces ${ }^{41}$ while shorter ligands bind to available sites. Neighboring CQD surfaces that have been stripped of oleate ligands but not yet replenished with new ligands are susceptible to necking (Supporting Information S1, S2). We sought to prevent this phenomenon following a recently reported strategy involving short thiol ligands introduced in solution. ${ }^{21}$ Since these thiols remain tightly bound on the surface during solid-state exchange, agglomeration can be prevented (Figure 3a, bottom).

Through density functional theory (DFT) modeling, we investigated how the liganding strategy affected the electronic landscape for carriers. The calculated DOS for a PbS quantum dot is shown in Figure $3 \mathrm{~b}$ (i). The states introduced by MPA are shown in panel (ii), and their peak is near the valence band edge of the
CQDs, thereby providing a reduced barrier to hole transport. On the other hand, propanethiol ligands introduce states only deep within the valence band and therefore militate against hole transport (panel (iii)). Thus, while they are able to prevent unwanted agglomeration, these ligands are expected to lead to poor interparticle conduction of carriers.

Interestingly, DFT also shows that the addition of a halide end group to the thiol ligand should prevent this problem. As shown in panel (iv), bromine-functionalized propanethiols introduce a large DOS near the valence band edge. Importantly, no detrimental midgap states arise from addition of these ligands to the quantum dot surface. DFT indicates that this bromothiol exchange should allow close proximity among CQDs while providing enough steric hindrance to prevent agglomeration, as shown in Figure 3a (bottom). While the DOS introduced by the bromothiol ligands is still over $1 \mathrm{eV}$ from the band edge, the reduction of the interparticle barrier to hole transport allows for improved coupling between adjacent quantum dots. This is demonstrated using a simple 1D calculation of the Schrödinger equation for proximate quantum wells with appropriate barrier heights (Supporting Information S3).

Guided by these indications from theory, we made films of CQDs using different surface passivation schemes and investigated their effects through optical and electrical studies. All films were cross-linked using an MPA solid-state exchange. X-ray diffraction was unable to resolve a significant difference in the films due to the small particle size and low overall amount of necking (Supporting Information S4). Thus, we investigated the steady-state photoluminescence $(\mathrm{PL})$ spectrum of each film (Figure 3c). One film had no solution-phase ligand treatment, one involved a hybrid passivation approach using $\mathrm{CdCl}_{2}$ solution-phase treatment, and the final film combined the $\mathrm{CdCl}_{2}$ treatment with a solution-phase bromothiol exchange.

All films exhibited a PL peak at approximately $1375 \mathrm{~nm}$, although the untreated film exhibited much weaker emission, suggestive of low photoluminescence quantum yield due to unpassivated surface traps. ${ }^{19}$ Both the untreated and $\mathrm{CdCl}_{2}$-only films displayed a broadened peak with a tail on the long-wavelength side. This is consistent with the existence of a small, but significant population of agglomerated CQDs that have a narrower band gap than the parent population. ${ }^{36}$ These agglomerates are optically active and thus are apparent in the PL spectra. The PL spectrum of the film with bromothiol ligands has a low-energy half-width at half-maximum approximately $20 \mathrm{meV}$ narrower, indicating that the ligands were able to suppress the fusion of CQDs into larger nanoparticles. A similar effect is seen in the long-wavelength tail of the film absorption spectra (Supporting Information S5), although the PL broadening is more apparent as carriers tend to be funneled into the smaller-band-gap inclusions. 
a
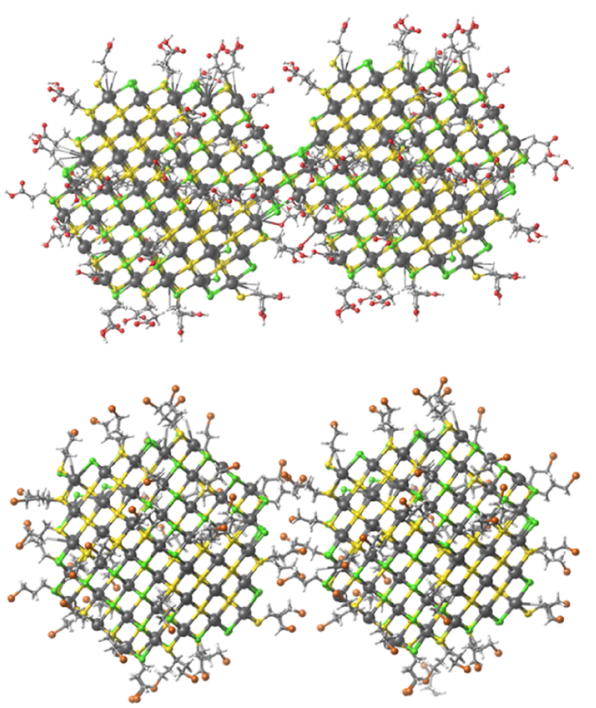

C

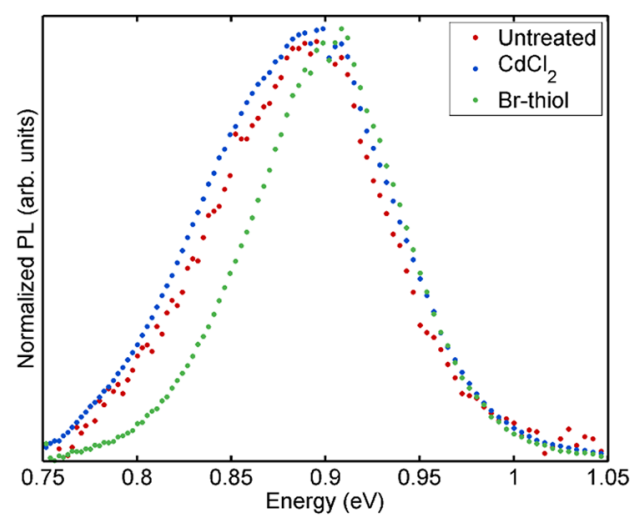

e

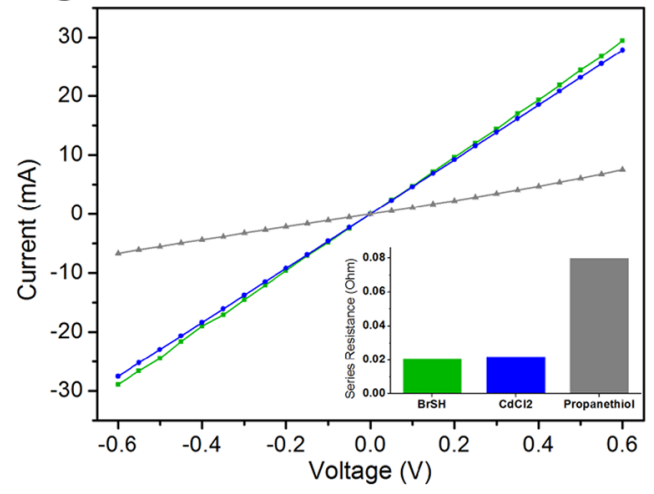

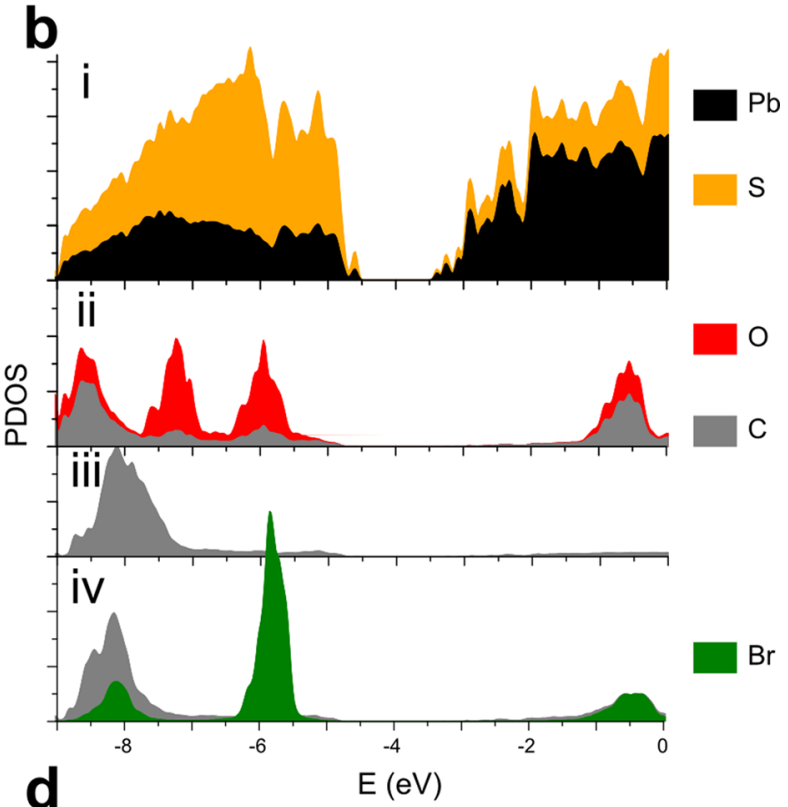

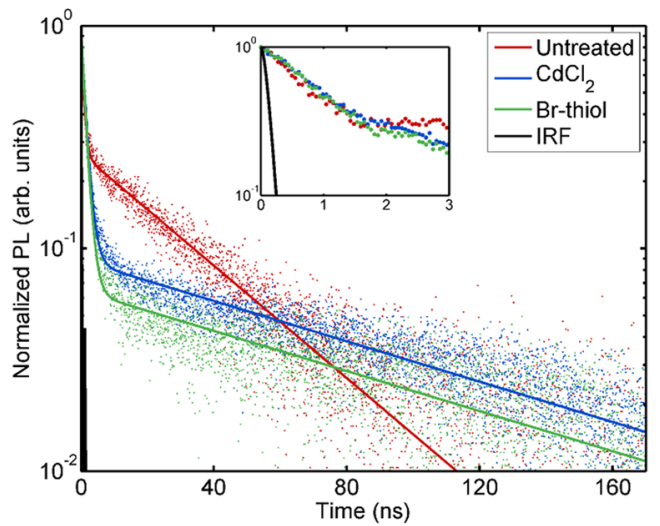

f

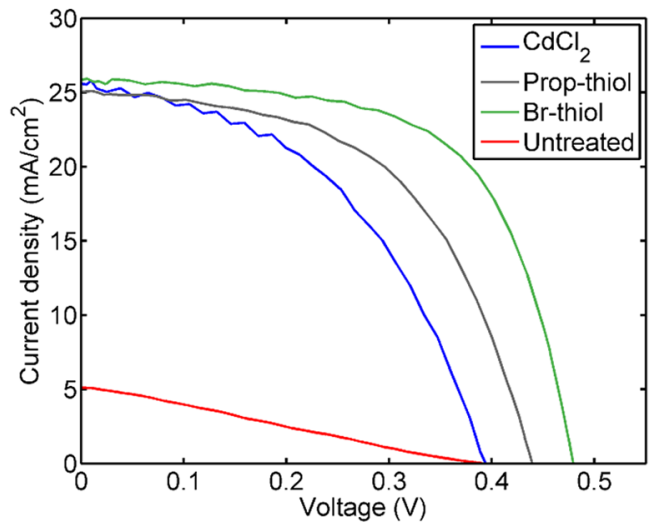

Figure 3. Effect of bromothiol ligands. (a) Top: CQDs can fuse during solid-state exchange, as surfaces are left temporarily uncovered. Bottom: The bromothiol ligands sterically prevent the necking of adjacent nanocrystals during solid-state ligand exchange. (b) DFT-calculated density of states for a ligand-covered CQD, separated into contributions from (i) the bare $\mathrm{PbS}$ quantum dot, (ii) MPA ligands, (iii) alkanethiol ligands, (iv) bromothiol ligands. (c) Solid-state PL comparing films with only MPA ligands (Untreated), hybrid passivation with added $\mathrm{CdCl}_{2}\left(\mathrm{CdCl}_{2}\right)$, and combined hybrid approach with bromothiol added in solution (Br-thiol). The absence of thiol ligands results in agglomeration, leading to optically active large nanocrystals exhibiting long-wavelength emission. (d) Transient photoluminescence decay spectroscopy of the same films, monitored at $1375 \mathrm{~nm}$ emission. Improved surface passivation through solution-phase $\mathrm{CdCl}_{2}$ addition leads to a reduction in surface trap associated decay dynamics. Inset shows short time scales to better resolve instrument response function (IRF). (e) Conductivity study comparing $\mathrm{CdCl}_{2}$-treated films without solution-added thiols, with propanethiols, and with bromothiols. Inset shows bar plot of device resistance. (f) $J-V$ curves of devices using different ligand schemes. 
Transient PL decay measurements were then carried out on each film, with emission monitored at the PL maximum. The decay dynamics are plotted in Figure 3d, and all traces are fit using a biexponential function. We follow the interpretation presented by Moroz et al. ${ }^{42}$ and Kholmicheva et al. ${ }^{43}$ in which the transient PL signature of a coupled nanocrystal film is assigned to two components. The fast decay component, $\tau_{1}$, is attributed to the dissociation of excitons and is therefore related to the ability of charges to move between neighboring CQDs. The slower component, $\tau_{2}$, is dominated by the recombination of free carriers through charge-trapping processes. A larger density of electronic trap sites thus increases the $\mathrm{PL}$ decay rate and reduces the magnitude of $\tau_{2}$.

The fast decay component of each film studied here has a time constant of approximately $1 \mathrm{~ns}$, indicating efficient dissociation of excitons in all of our films. The longer, trap-associated decay component, $\tau_{2}$, combines the effects of radiative recombination $(\sim 1 \mu \mathrm{s})$ and surface trapping processes, which accelerate the decay. Notably, $\tau_{2}$ for the MPA-only film (untreated) is $34.4 \mathrm{~ns}$, while the inclusion of $\mathrm{CdCl}_{2}$, both with and without Br-thiol, increases this value to 95.9 and $96.4 \mathrm{~ns}$, respectively. This confirms the surface passivation effect of added $\mathrm{CdCl}_{2}$ in reducing nonradiative recombination of CQD films. The relative PL brightness supports this view, with the presence of $\mathrm{CdCl}_{2}$ leading to a large increase in signal strength (Supporting Information S6).

These PL studies reveal the benefits of a combined ligand strategy incorporating bromothiols with a hybrid passivation approach. The MPA- $\mathrm{CdCl}_{2}$ treatment provides effective passivation of surface trap sites but is unable to prevent CQD fusion. Adding thiol ligands in solution prevents the formation of quantum traps and, combined with $\mathrm{CdCl}_{2}$ and MPA, produces the best available passivation.

We probed the electronic transport properties of our films using conductivity measurements on films. We developed test structures with symmetric holeextracting contacts (i.e., Au and PEDOT:PSS/ITO), with the results shown in Figure $3 \mathrm{e}$. Hybrid $\mathrm{CdCl}_{2}$ films showed a high conductivity of $8.4 \times 10^{-3} \Omega^{-1} \mathrm{~cm}^{-1}$ due to strong coupling from MPA ligands and potentially reduced interparticle barriers due to CQD fusion. Using propanethiol reduced the conductivity 4-fold to $2.2 \times 10^{-3} \Omega^{-1} \mathrm{~cm}^{-1}$. We propose that the replacement of MPA surface sites by the insulating alkane chains leads to a reduction in their DOS near the valence band edge and a greater barrier to hole transfer between CQDs. Notably, the use of Br-thiol ligands-with the added valence band associated states-allowed the film to match the conductivity of the hybrid passivated film at $9.0 \times 10^{-3} \Omega^{-1} \mathrm{~cm}^{-1}$ while simultaneously preventing fusing of dots as indicated by the narrower, bluer PL seen

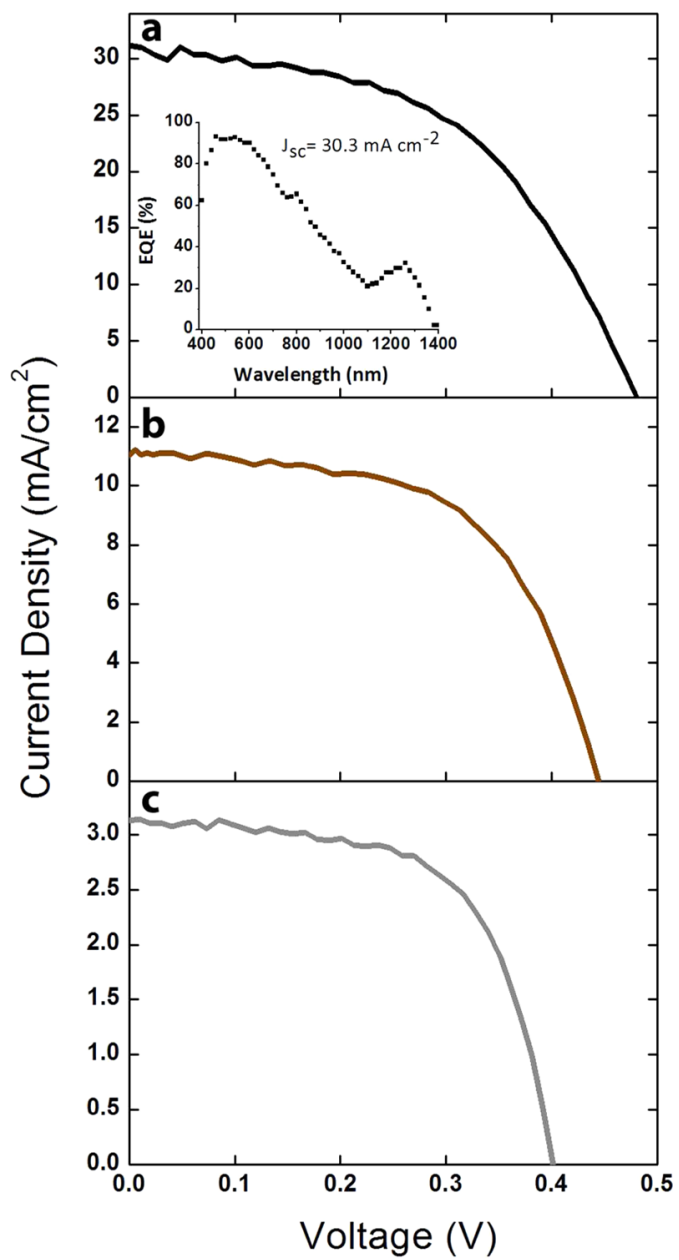

Figure 4. Measured current-voltage characteristics of a $\mathrm{Br}$-thiol/ $\mathrm{CdCl}_{2}$ treated PbS QD device (a) under AM1.5 simulated solar illumination, (b) after using a perovskite solar cell representative filter (long-pass $750 \mathrm{~nm}$ filter), and (c) after using a silicon solar cell representative filter (longpass $1100 \mathrm{~nm}$ filter). Inset: EQE curve for an unfiltered device. The integrated current value is also shown.

in Figure 3c. To ensure that the difference in conductivities was not due to our ligand approaches changing the doping characteristics of our films, we also performed capacitance-voltage measurements. These revealed that the charge carrier densities were unchanged among the samples (Supporting Information S7).

The PV performance of our CQD films was assessed under AM1.5-simulated solar illumination. The current density-voltage $(J-V)$ curves for devices made using different ligand strategies are shown in Figure $3 f$. Without $\mathrm{CdCl}_{2}$ or thiol treatment, devices performed very poorly due to the high density of surface trap states. While the hybrid $\mathrm{CdCl}_{2}$ device displayed high short-circuit current $\left(J_{\mathrm{SC}}\right)$, the open-circuit voltage $\left(V_{\mathrm{OC}}\right)$ was quite low, as expected from the PL-proven existence of agglomerated nanoparticles. The fill factor was also low, likely due to poor extraction of carriers that are trapped in these small-band-gap inclusions. Addition of propanethiol ligands to prevent agglomeration increased the $V_{\mathrm{OC}}$, but with a slight reduction in 
$J_{\mathrm{SC}}$ and a fill factor below 0.5 . The use of $\mathrm{Br}$-thiol ligands instead resulted in an increase in the fill factor to over 0.6 , as well as a further increase in $V_{\mathrm{OC}}$ without any reduction of Jsc. This led to a PCE exceeding $7 \%$. Thus, the combined ligand strategy enabled us to produce high-efficiency solar cells using small-band-gap CQD absorbers.

We applied the optimized approach to devices illuminated using filtered AM1.5, thereby mimicking the effect of absorption by a front wide-band-gap solar cell. We used long wave pass (LWP) filters to emulate either a perovskite (750 nm LWP) or silicon (1100 nm LWP) front cell and calculated a mismatch factor to account for differences between our lamp and AM1.5 spectra. Figure 4 shows the $J-V$ characteristics of the device under unfiltered (Figure 4a), perovskitefiltered (Figure 4b), and silicon-filtered (Figure 4c) AM1.5 light. The device employed the full ligand strategy using bromothiols- and $\mathrm{CdCl}_{2}$-treated $1300 \mathrm{~nm}$ PbS CQDs as the absorber. The representative $V_{\mathrm{OC}}, J_{\mathrm{SC}}$, fill factor (FF), and PCE results are shown in Table 1. The reported 7.3\% PCE for the unfiltered case is the best AM1.5 performance reported for any solution-processed material with a band gap narrower than $1 \mathrm{eV}$. Interestingly, the loss of $V_{\mathrm{OC}}$, referenced to the CQDs' band gap, decreases as the band gap of the nanocrystal decreases (i.e., $E_{\mathrm{g}}-q V_{\mathrm{OC}} \cong$ $0.5 \mathrm{eV}$, compared with $\cong 0.7 \mathrm{eV}$ for $1.3 \mathrm{eV}$, or $950 \mathrm{~nm}$, CQDs). We attribute this improvement to the reduced conduction band offset. An external quantum efficiency curve is shown as the inset for Figure 4 that demonstrates a broad spectral response and an integrated current density of $30.3 \mathrm{~mA} \mathrm{~cm}{ }^{-2}$, which matches well with the value reported under AM1.5 solar simulator.

Filtering the device leads to $2.8 \%$ PCE after $750 \mathrm{~nm}$ LWP filter (perovskite PV) and 0.8\% PCE after $1100 \mathrm{~nm}$ LWP filter (silicon PV), with the results shown in Table 1.

An addition of 2.8 power points to the perovskite cell power conversion efficiency that recently soared to
TABLE 1. Open-Circuit Voltage $\left(V_{o c}\right)$, Short-Circuit Current Density $\left(J_{S C}\right)$, Fill Factor (FF), and Power Conversion Efficiency (PCE) Results for Unfiltered, Perovskite-Filtered, and Silicon-Filtered Devices

\begin{tabular}{lcccc}
\multicolumn{1}{c}{ type } & $\boldsymbol{V}_{\mathbf{0 C}}(\mathbf{V})$ & $\boldsymbol{J}_{\mathbf{S C}}\left(\mathbf{m A ~ c m}^{-\mathbf{2}}\right)$ & FF (\%) & PCE (\%) \\
\hline unfiltered & 0.48 & $31 \pm 1$ & 52 & 7.3 \\
perovskite filtered $(750 \mathrm{~nm})$ & 0.45 & $11 \pm 1$ & 57 & 2.8 \\
silicon filtered $(1100 \mathrm{~nm})$ & 0.40 & $3.0 \pm 0.2$ & 61 & 0.8 \\
\hline
\end{tabular}

over $20 \%$ certified $\mathrm{PCE}^{8}$ is of potential applied interest. Silicon PV technology is sufficiently mature that an improvement of 0.8 power point over the best $\mathrm{Si}$ cells is also attractive. ${ }^{44}$ If the 6 power points in principle available to crystalline $\mathrm{Si}$ cells could be more closely approached, this enhancement could significantly impact power generation given the ubiquity of silicon solar cells.

\section{CONCLUSION}

In sum, we have demonstrated the utility of CQDs as an infrared absorber that can enhance the performance of high-efficiency PV materials. Detailed balance modeling was used to select a suitable optical band gap for use with a wide range of systems, and DFT calculations were used to develop a multiple-ligand strategy that prevented agglomeration of adjacent dots while facilitating carrier transport. Optical studies of the film confirmed that the ligand approach prevented development of two sources of electronic traps: unpassivated surface sites and small-band-gap CQD agglomerates. Conductivity measurements confirmed that the bromine-functionalized thiols are well-coupled. Depleted heterojunction devices were fabricated and achieved the highest single-junction efficiency for a work suggests access to significant enhancements in the solar harvesting ability of the highest-performing photovoltaic systems with the addition of a low-cost, solution-processed quantum dot absorber. $<1.0 \mathrm{eV}$ band-gap solution-processed solar cell. The

to $140{ }^{\circ} \mathrm{C}$ under argon, followed by the swift injection of the sulfur precursor of $0.210 \mathrm{~mL}$ of hexamethyldisilathiane (TMS) and $8.0 \mathrm{~mL}$ of 1-octadecene. $\mathrm{CdCl}_{2}$ precursor preparation was described previously. ${ }^{19}$ Bromopropanethiol (Beyond Pharmaceutical Co.) or propanethiol (Sigma-Aldrich) was mixed with 1-octadecene to a concentration of $0.5 \mathrm{mmol}$ per batch. Thiol and/or $\mathrm{CdCl}_{2}$ treatments were performed during the cooling process following previously published methods, ${ }^{19,21}$ with precursor concentrations of 0.5 and $0.3 \mathrm{mmol} \mathrm{mL}^{-1}$, respectively. After synthesis, the PbS dots were purified and extracted two times with $50 \mathrm{~mL}$ of distilled acetone and redispersed in toluene. Two washes using methanol were performed before final redispersion at $50 \mathrm{mg} \mathrm{mL}^{-1}$ in octane.

Photovoltaic Device Fabrication. Unpatterned and prepatterned ITO-coated glass substrates were purchased from Delta Technologies and TFD Inc., respectively. $\mathrm{TiO}_{2}$ nanoparticles were synthesized based on a modified solvothermal method previously reported. ${ }^{30}$ Briefly, $\mathrm{TiCl}_{4}$ (Sigma-Aldrich) was added 
dropwise to ethanol to a 1:4 volumetric ratio. A 2 mol \% amount of $\mathrm{NbCl}_{5}$ (Sigma-Aldrich) was dissolved in the resulting solution. This solution was added to benzyl alcohol in a 1:8 volumetric ratio, upon which the mixture turned red. The jar was sealed and placed in an oven at $70^{\circ} \mathrm{C}$ overnight $(18 \mathrm{~h})$. The resulting solution was slightly hazy but not opaque. The $\mathrm{TiO}_{2} \mathrm{NPs}$ were precipitated and washed twice using diethyl ether. The resulting powder was redispersed in methanol at a $10 \mathrm{mg} \mathrm{mL}^{-1}$ concentration and sonicated until uniform. Polyethylenimine ethoxylated was purchased from Sigma-Aldrich and diluted 100 times in methanol.

The diluted PEIE solution was spin-cast onto the ITO substrates at $2500 \mathrm{rpm}$ for $10 \mathrm{~s}$. Two layers of $\mathrm{TiO}_{2}$ nanoparticles were then deposited by spin-coating $\sim 75 \mu \mathrm{L}$ of solution at $2500 \mathrm{rpm}$ for $10 \mathrm{~s}$ each. Following deposition of the $\mathrm{PEIE} / \mathrm{TiO}_{2}$ electrodes, the substrates were dried on a hot plate in air for $30 \mathrm{~min}$ at $100^{\circ} \mathrm{C}$

PbS QD films were deposited using a layer-by-layer spincoating process under ambient conditions. Two drops of filtered CQD solution (50 $\mathrm{mg} \mathrm{mL}^{-1}$ in octane) was deposited on the $\mathrm{PEIE} / \mathrm{TiO}_{2}$-coated ITO/glass substrate and spin-cast at $2500 \mathrm{rpm}$ for $10 \mathrm{~s}$ to form each layer. Solid-state ligand exchange was performed using a 5\% MPA solution in methanol and spinning at $2500 \mathrm{rpm}$ for drying after $3 \mathrm{~s}$ of soaking. The QD film was washed two times with methanol to remove excess MPA and unbound oleate ligands. Top contacts were deposited using an Angstrom Engineering Åmod deposition system in an Innovative Technology glovebox. The contact consists of approximately $40 \mathrm{~nm}$ of thermally evaporated molybdenum oxide, $50 \mathrm{~nm}$ of electron-beam deposited gold, and approximately $100 \mathrm{~nm}$ of thermally evaporated silver.

Scanning Electron Microscopy. Scanning electron microscopy (SEM) imaging was performed at a pressure of $6.3 \times 10^{-4} \mathrm{~Pa}$ using an FEI Quanta Environmental SEM at $15 \mathrm{kV}$ using a secondary electron detector and a working distance of $10 \mathrm{~mm}$.

DFT Calculation. DFT calculations were done by utilizing a dual basis of localized Gaussians and plane waves in the Quickstep module of the CP2K program suite. ${ }^{46,47}$ Goedecker-Teter-Hutter pseudopotentials ${ }^{48}$ were employed with an appropriate 300 Ry grid cutoff. To reduce the basis set superposition errors in molecules, a localized basis set of double- $\zeta$ plus polarization (DZVP) was used. ${ }^{49}$ The Perdew-Burke-Ernzerhof (PBE) exchange correlation functional was used for the calculations, and when forces were less than $40 \mathrm{meV} / \AA$, the structures were considered relaxed. Simulations were done for $2.4 \mathrm{~nm}$ quantum dots in a $50 \times 50 \times 50 \AA^{3}$ unit cell. The quantum dot was carved out of bulk PbS where all singly bonded atoms were discarded, yielding a faceted cuboctahedron shape. A mixture of $\mathrm{Cl}$ and thiol ligands (propanethiol, bromopropanethiol, MPA) was used to passivate all dangling bonds on (111) and (100) facets. Stoichiometry was selected such that the charge neutrality of the dot is preserved, which is necessary to position the Fermi level in the midgap. ${ }^{50}$

The input files are provided in the SI.

Photoluminescence. Spectral and transient photoluminescence measurements were carried out using a Horiba FluoroLog-3 spectrofluorometer in reflection geometry under ambient conditions. The sample was excited using a $633 \mathrm{~nm}$ pulsed laser diode ( $<1 \mathrm{~ns}$ ). The emission was passed through a $1000 \mathrm{~nm}$ blaze grating monochromator (iHR320) and collected by an infrared photomultiplier tube.

AM1.5 Photovoltaic Performance Characterization. Current-voltage characteristics were measured using a Keithley 2400 source meter. The devices were tested under $\mathrm{N}_{2}$ flow. The solar spectrum at AM1.5 was simulated to within class A specification (less than $25 \%$ spectral mismatch) with a xenon lamp and filters (ScienceTech; measured intensity of $100 \mathrm{~mW} \mathrm{~cm}^{-2}$ ). The source intensity was measured using a Melles-Griot broadband power meter through a circular aperture of $0.049 \mathrm{~cm}^{2}$ at the position of the device and was confirmed by a calibrated reference solar cell (Newport). The accuracy of current-voltage measurements is estimated to be $\pm 7 \%$. Filters (750 and $1100 \mathrm{~nm}$ long-pass) from Thorlabs, Inc. were used to represent a perovskite solar cell and a silicon solar cell, respectively.
Spectral Mismatch Calculation. The spectral power within the wavelength region of interest was calibrated using the lamp spectrum supplied by the manufacturer. The absolute power of the lamp spectrum was calibrated with a calibrated power meter and a set of calibrated long-pass filters. The external quantum efficiency (EQE) spectrum of the $1.3 \mu \mathrm{m}$ colloidal quantum dot solar cell device was measured and used to calculate the mismatch factor between the lamp spectrum filtered with a $1100 \mathrm{~nm}$ long-pass filter (1100 LPF) and the AM1.5 spectrum beyond $1100 \mathrm{~nm}$. The spectral mismatch was calculated with the ratio of the integral of the product of the lamp spectrum, the EQE spectrum, and 1100LPF spectrum and the integral of the product of the AM1.5 spectrum, the EQE spectrum, and 1100 LPF spectrum. This resulted in a mismatch factor of 1.5. The main error in the mismatch factor is estimated to be in the calibration of the lamp spectrum, estimated to be about $10 \%$.

EQE Measurement. External quantum efficiency measurements were obtained by applying chopped $(220 \mathrm{~Hz})$ monochromatic illumination, collimated and cofocused with a 0.7 Sun intensity white light source, on the device of interest. To create monochromatic illumination, a $450 \mathrm{~W}$ xenon lamp was used, which passes through a monochromator with order-sorting filters. Calibrated Newport 818-UV and Newport 818-IR power meters were used to measure power. The response from the chopped signal was measured using a Stanford Research system current preamplifier feeding into a Stanford Research system lock-in amplifier set to voltage mode. The uncertainty in the EQE measurements was estimated to be $3 \%$.

Conflict of Interest: The authors declare no competing financial interest.

Acknowledgment. This publication was based in part on work supported by Award KUS-11-009-21, made by King Abdullah University of Science and Technology (KAUST), by the Ontario Research Fund Research Excellence Program, and by the Natural Sciences and Engineering Research Council (NSERC) of Canada. This research is supported in part by the IBM Canada Research and Development Center. Computations were performed on the GPC supercomputer at the SciNet HPC Consortium. SciNet is funded by the Canada Foundation for Innovation under the auspices of Compute Canada; the Government of Ontario; Ontario Research Fund - Research Excellence; and the University of Toronto. The authors would like to thank R. Comin, G. Walters, E. Yassitepe, F. P. G. de Arquer, Z. Yang, A. Labelle, L. Rollny, D. Zhitomirsky, and G. Moreno-Bautista for their help throughout the course of this study.

Supporting Information Available: The Supporting Information is available free of charge on the ACS Publications website at DOI: 10.1021/acsnano.5b02164.

Nine supplementary images demonstrating the effect of aggregation and the influence of surface ligands on optical and electronic properties (PDF)

Source code for DFT simulations (TXT)

\section{REFERENCES AND NOTES}

1. Jean, J.; Brown, P. R.; Jaffe, R. L.; Buonassisi, T.; Bulović, V. Pathways for Solar Photovoltaics. Energy Environ. Sci. 2015, 8, 1200.

2. Green, M. A.; Emery, K.; Hishikawa, Y.; Warta, W.; Dunlop, E. D. Solar Cell Efficiency Tables (Version 45). Prog. Photovoltaics 2015, 23, 1-9.

3. Li, G.; Zhu, R.; Yang, Y. Polymer Solar Cells. Nat. Photonics 2012, 6, 153-161.

4. Grätzel, M. Dye-Sensitized Solar Cells. J. Photochem. Photobiol., C 2003, 4, 145-153.

5. Lee, M. M.; Teuscher, J.; Miyasaka, T.; Murakami, T. N.; Snaith, H. J. Efficient Hybrid Solar Cells Based on MesoSuperstructured Organometal Halide Perovskites. Science 2012, 338, 643-647.

6. Kim, H.-S.; Lee, C.-R.; Im, J.-H.; Lee, K.-B.; Moehl, T.; Marchioro, A.; Moon, S.-J.; Humphry-Baker, R.; Yum, J.-H.; Moser, J. E.; et al. Lead lodide Perovskite Sensitized All-Solid-State Submicron Thin Film Mesoscopic Solar 
Cell with Efficiency Exceeding 9\%. Sci. Rep. 2012, 2. DOI: 10.1038/srep00591

7. Jeon, N. J.; Noh, J. H.; Yang, W. S.; Kim, Y. C.; Ryu, S.; Seo, J.; Seok, S. I. Compositional Engineering of Perovskite Materials for High-Performance Solar Cells. Nature 2015, 517, 476-480.

8. Yang, W. S.; Noh, J. H.; Jeon, N. J.; Kim, Y. C.; Ryu, S.; Seo, J.; Seok, S. I. High-Performance Photovoltaic Perovskite Layers Fabricated through Intramolecular Exchange. Science 2015, 348 , aaa9272.

9. Sargent, E. H. Infrared Photovoltaics Made by Solution Processing. Nat. Photonics 2009, 3, 325-331.

10. Cotal, H.; Fetzer, C.; Boisvert, J.; Kinsey, G.; King, R.; Hebert, P.; Yoon, H.; Karam, N. III-V Multijunction Solar Cells for Concentrating Photovoltaics. Energy Environ. Sci. 2009, 2, 174-192.

11. Li, W.; Hendriks, K. H.; Roelofs, W. S. C.; Kim, Y.; Wienk, M. M.; Janssen, R. A. J. Efficient Small Bandgap Polymer Solar Cells with High Fill Factors for $300 \mathrm{Nm}$ Thick Films. Adv. Mater. 2013, 25, 3182-3186.

12. Mathew, S.; Yella, A.; Gao, P.; Humphry-Baker, R.; Curchod, B. F. E.; Ashari-Astani, N.; Tavernelli, I.; Rothlisberger, U.; Nazeeruddin, M. K.; Grätzel, M. Dye-Sensitized Solar Cells with 13\% Efficiency Achieved through the Molecular Engineering of Porphyrin Sensitizers. Nat. Chem. 2014, 6, 242-247.

13. Eperon, G. E.; Stranks, S. D.; Menelaou, C.; Johnston, M. B.; Herz, L. M.; Snaith, H. J. Formamidinium Lead Trihalide: A Broadly Tunable Perovskite for Efficient Planar Heterojunction Solar Cells. Energy Environ. Sci. 2014, 7, 982-988.

14. Lee, J.-W.; Seol, D.-J.; Cho, A.-N.; Park, N.-G. High-Efficiency Perovskite Solar Cells Based on the Black Polymorph of HC(NH2)2Pbl3. Adv. Mater. 2014, 26, 4991-4998.

15. Wang, X.; Koleilat, G. I.; Tang, J.; Liu, H.; Kramer, I. J.; Debnath, R.; Brzozowski, L.; Barkhouse, D. A. R.; Levina, L.; Hoogland, S.; et al. Tandem Colloidal Quantum Dot Solar Cells Employing a Graded Recombination Layer. Nat. Photonics 2011, 5, 480-484.

16. Kramer, I. J.; Minor, J. C.; Moreno-Bautista, G.; Rollny, L.; Kanjanaboos, P.; Kopilovic, D.; Thon, S. M.; Carey, G. H.; Chou, K. W.; Zhitomirsky, D.; et al. Efficient Spray-Coated Colloidal Quantum Dot Solar Cells. Adv. Mater. 2015, 27, 116-121.

17. Kramer, I. J.; Moreno-Bautista, G.; Minor, J. C.; Kopilovic, D.; Sargent, E. H. Colloidal Quantum Dot Solar Cells on Curved and Flexible Substrates. Appl. Phys. Lett. 2014, 105, 163902.

18. Tang, J.; Kemp, K. W.; Hoogland, S.; Jeong, K. S.; Liu, H.; Levina, L.; Furukawa, M.; Wang, X.; Debnath, R.; Cha, D.; et al. Colloidal-Quantum-Dot Photovoltaics Using AtomicLigand Passivation. Nat. Mater. 2011, 10, 765-771.

19. Ip, A. H.; Thon, S. M.; Hoogland, S.; Voznyy, O.; Zhitomirsky, D.; Debnath, R.; Levina, L.; Rollny, L. R.; Carey, G. H.; Fischer, A.; et al. Hybrid Passivated Colloidal Quantum Dot Solids. Nat. Nanotechnol. 2012, 7, 577-582.

20. Ning, Z.; Voznyy, O.; Pan, J.; Hoogland, S.; Adinolfi, V.; Xu, J.; Li, M.; Kirmani, A. R.; Sun, J.-P.; Minor, J.; et al. Air-Stable N-Type Colloidal Quantum Dot Solids. Nat. Mater. 2014, 13, 822-828.

21. Zhitomirsky, D.; Voznyy, O.; Levina, L.; Hoogland, S.; Kemp, K. W.; Ip, A. H.; Thon, S. M.; Sargent, E. H. Engineering Colloidal Quantum Dot Solids within and beyond the Mobility-Invariant Regime. Nat. Commun. 20145 DOI: 10.1038/ncomms4803

22. Lan, X.; Bai, J.; Masala, S.; Thon, S. M.; Ren, Y.; Kramer, I. J.; Hoogland, S.; Simchi, A.; Koleilat, G. I.; Paz-Soldan, D.; et al. Self-Assembled, Nanowire Network Electrodes for Depleted Bulk Heterojunction Solar Cells. Adv. Mater. 2013, $25,1769-1773$

23. Paz-Soldan, D.; Lee, A.; Thon, S. M.; Adachi, M. M.; Dong, H.; Maraghechi, P.; Yuan, M.; Labelle, A. J.; Hoogland, S.; Liu, K.; et al. Jointly Tuned Plasmonic-Excitonic Photovoltaics Using Nanoshells. Nano Lett. 2013, 130306104227004.

24. Labelle, A. J.; Thon, S. M.; Masala, S.; Adachi, M. M.; Dong, H.; Farahani, M.; Ip, A. H.; Fratalocchi, A.; Sargent, E. H. Colloidal Quantum Dot Solar Cells Exploiting Hierarchical Structuring. Nano Lett. 2015, 15, 1101-1108.
25. Chuang, C.-H. M.; Brown, P. R.; Bulović, V.; Bawendi, M. G. Improved Performance and Stability in Quantum Dot Solar Cells through Band Alignment Engineering. Nat. Mater. 2014, 13, 796-801.

26. Lan, X.; Voznyy, O.; Kiani, A.; de Arquer, F. P. G.; Abbas, A. S.; Kim, G.-H.; Liu, M.; Yang, Z.; Walters, G.; Xu, J.; et al., Passivation Using Molecular Halides Increases Quantum Dot Solar Cell Performance. Adv. Mater. 2015, Submitted for Publication.

27. Chen, C.-C.; Chang, W.-H.; Yoshimura, K.; Ohya, K.; You, J.; Gao, J.; Hong, Z.; Yang, Y. An Efficient Triple-Junction Polymer Solar Cell Having a Power Conversion Efficiency Exceeding 11\%. Adv. Mater. 2014, 26, 5670-5677.

28. Yusoff, A. R. b. M.; Kim, D.; Kim, H. P.; Shneider, F. K.; da Silva, W. J.; Jang, J. A High Efficiency Solution Processed Polymer Inverted Triple-Junction Solar Cell Exhibiting a Power Conversion Efficiency of 11.83\%. Energy Environ. Sci. 2015. 8, 303-316.

29. Pattantyus-Abraham, A. G.; Kramer, I. J.; Barkhouse, A. R.; Wang, X.; Konstantatos, G.; Debnath, R.; Levina, L.; Raabe, I.; Nazeeruddin, M. K.; Grätzel, M. Depleted-Heterojunction Colloidal Quantum Dot Solar Cells. ACS Nano 2010, 4, 3374-3380.

30. Niederberger, M.; Bartl, M. H.; Stucky, G. D. Benzyl Alcohol and Titanium TetrachlorideA Versatile Reaction System for the Nonaqueous and Low-Temperature Preparation of Crystalline and Luminescent Titania Nanoparticles. Chem. Mater. 2002, 14, 4364-4370.

31. Zhou, Y.; Fuentes-Hernandez, C.; Shim, J.; Meyer, J.; Giordano, A. J.; Li, H.; Winget, P.; Papadopoulos, T.; Cheun, H.; Kim, J.; et al. A Universal Method to Produce Low-Work Function Electrodes for Organic Electronics. Science 2012, 336, 327-332.

32. Hyun, B.-R.; Zhong, Y.-W.; Bartnik, A. C.; Sun, L.; Abruña, H. D.; Wise, F. W.; Goodreau, J. D.; Matthews, J. R.; Leslie, T. M.; Borrelli, N. F. Electron Injection from Colloidal PbS Quantum Dots into Titanium Dioxide Nanoparticles. ACS Nano 2008, 2, 2206-2212.

33. Sambur, J. B.; Riha, S. C.; Choi, D.; Parkinson, B. A. Influence of Surface Chemistry on the Binding and Electronic Coupling of CdSe Quantum Dots to Single Crystal TiO2 Surfaces. Langmuir 2010, 26, 4839-4847.

34. Hanrath, T.; Veldman, D.; Choi, J. J.; Christova, C. G.; Wienk, M. M.; Janssen, R. A. J. PbSe Nanocrystal Network Formation during Pyridine Ligand Displacement. ACS Appl. Mater. Interfaces 2009, 1, 244-250.

35. Dong, $A_{\text {.; }}$ Ye, $X_{\text {.; }}$ Chen, J.; Kang, Y.; Gordon, T.; Kikkawa, J. M.; Murray, C. B. A Generalized Ligand-Exchange Strategy Enabling Sequential Surface Functionalization of Colloidal Nanocrystals. J. Am. Chem. Soc. 2011, 133, 998-1006.

36. Carey, G. H.; Levina, L.; Comin, R.; Voznyy, O.; Sargent, E. H. Record Charge Carrier Diffusion Length in Colloidal Quantum Dot Solids via Mutual Dot-To-Dot Surface Passivation. Adv. Mater. 2015, 27, 3325-3330.

37. Oh, S. J.; Berry, N. E.; Choi, J.-H.; Gaulding, E. A.; Paik, T.; Hong, S.-H.; Murray, C. B.; Kagan, C. R. Stoichiometric Control of Lead Chalcogenide Nanocrystal Solids to Enhance Their Electronic and Optoelectronic Device Performance. ACS Nano 2013, 7, 2413-2421.

38. Liu, Y.; Tolentino, J.; Gibbs, M.; Ihly, R.; Perkins, C. L.; Liu, Y.; Crawford, N.; Hemminger, J. C.; Law, M. PbSe Quantum Dot Field-Effect Transistors with Air-Stable Electron Mobilities above $7 \mathrm{~cm}^{2} \mathrm{~V}^{-1} \mathrm{~s}^{-1}$. Nano Lett. 2013, 13, 1578-1587.

39. Baumgardner, W. J.; Whitham, K.; Hanrath, T. Confinedbut-Connected Quantum Solids via Controlled Ligand Displacement. Nano Lett. 2013, 13, 3225-3231.

40. Boneschanscher, M.P.; Evers, W.H.; Geuchies, J. J.; Altantzis, T.; Goris, B.; Rabouw, F. T.; van Rossum, S. A. P.; van der Zant, H. S. J.; Siebbeles, L. D. A.; Tendeloo, G. V.; et al. Long-Range Orientation and Atomic Attachment of Nanocrystals in 2D Honeycomb Superlattices. Science 2014, 344, 1377-1380.

41. Hassinen, A.; Moreels, I.; De Nolf, K.; Smet, P. F.; Martins, J. C.; Hens, Z. Short-Chain Alcohols Strip X-Type Ligands and Quench the Luminescence of PbSe and CdSe Quantum Dots, Acetonitrile Does Not. J. Am. Chem. Soc. 2012, 134, 20705-20712. 
42. Moroz, P.; Kholmicheva, N.; Mellott, B.; Liyanage, G.; Rijal, U.; Bastola, E.; Huband, K.; Khon, E.; McBride, K.; Zamkov, M. Suppressed Carrier Scattering in CdS-Encapsulated PbS Nanocrystal Films. ACS Nano 2013, 7, 6964-6977.

43. Kholmicheva, N.; Moroz, P.; Bastola, E.; Razgoniaeva, N.; Bocanegra, J.; Shaughnessy, M.; Porach, Z.; Khon, D.; Zamkov, M. Mapping the Exciton Diffusion in Semiconductor Nanocrystal Solids. ACS Nano 2015, 9, 2926-2937.

44. Green, M. A. The Path to $25 \%$ Silicon Solar Cell Efficiency: History of Silicon Cell Evolution. Prog. Photovoltaics 2009, 17, 183-189.

45. Henry, C. H. Limiting Efficiencies of Ideal Single and Multiple Energy Gap Terrestrial Solar Cells. J. Appl. Phys. 1980, 51, 4494-4500.

46. VandeVondele, J.; Krack, M.; Mohamed, F.; Parrinello, M.; Chassaing, T.; Hutter, J. Quickstep: Fast and Accurate Density Functional Calculations Using a Mixed Gaussian and Plane Waves Approach. Comput. Phys. Commun. 2005, 167, 103-128.

47. Lippert, B. G.; Hutter, J.; Parrinello, M. A Hybrid Gaussian and Plane Wave Density Functional Scheme. Mol. Phys. 1997, 92, 477-488.

48. Hartwigsen, C.; Goedecker, S.; Hutter, J. Relativistic Separable Dual-Space Gaussian Pseudopotentials from $\mathrm{H}$ to $\mathrm{Rn}$. Phys. Rev. B 1998, 58, 3641-3662.

49. VandeVondele, J.; Hutter, J. Gaussian Basis Sets for Accurate Calculations on Molecular Systems in Gas and Condensed Phases. J. Chem. Phys. 2007, 127, 114105.

50. Voznyy, O.; Zhitomirsky, D.; Stadler, P.; Ning, Z.; Hoogland, S.; Sargent, E. H. A Charge-Orbital Balance Picture of Doping in Colloidal Quantum Dot Solids. ACS Nano 2012, 6, 8448-8455. 